Published in final edited form as:

Neurotox Res. 2020 February ; 37(2): 326-337. doi:10.1007/s12640-019-00133-8.

\title{
Comparing the Neuroprotective Effects of Caffeic Acid in Rat Cortical Slices and Caenorhabditis elegans: Involvement of Nrf2 and SKN-1 Signaling Pathways
}

\author{
Aline Colonnello ${ }^{1,2}$, Gabriela Aguilera-Portillo ${ }^{1}$, Leonardo C. Rubio-López ${ }^{1,2}$, Benjamín \\ Robles-Bañuelos ${ }^{1}$, Edgar Rangel-López ${ }^{1}$, Samaria Cortez-Núñez ${ }^{3}$, Yadira Evaristo-Priego ${ }^{3}$, \\ Alejandro Silva-Palacios ${ }^{4}$, Sonia Galván-Arzate ${ }^{5}$, Rodolfo García-Contreras ${ }^{6}$, Isaac \\ Túnez $^{7,8}$, Pan Chen $^{9}$, Michael Ashner ${ }^{9}$, Abel Santamaría ${ }^{1,{ }^{*}}$ \\ ${ }^{1}$ Laboratorio de Aminoácidos Excitadores, Instituto Nacional de Neurología y Neurocirugía, \\ Mexico City, Mexico. \\ ${ }^{2}$ Facultad de Ciencias, Universidad Nacional Autónoma de México, Mexico City, Mexico. \\ ${ }^{3}$ Escuela Superior de Ciencias Naturales, Universidad Autónoma de Guerrero, Chilpancingo, \\ Guerrero, Mexico. \\ ${ }^{4}$ Departamento de Biomedicina Cardiovascular, Instituto Nacional de Cardiología Ignacio Chávez, \\ Mexico City, Mexico. \\ ${ }^{5}$ Departamento de Neuroquímica, Instituto Nacional de Neurología y Neurocirugía, Mexico City, \\ Mexico. \\ ${ }^{6}$ Departamento de Microbiología y Parasitología, Facultad de Medicina, Universidad Nacional \\ Autónoma de México, Mexico City, Mexico. \\ ${ }^{7}$ Departamento de Bioquímica y Biología Molecular, Facultad de Medicina y Enfermería, \\ Universidad de Córdoba, Cordoba, Spain. \\ ${ }^{8}$ Instituto Maimonides de Investigación Biomédica de Córdoba (IMIBIC), Cordoba, Spain. \\ ${ }^{9}$ Albert Einstein College of Medicine, Jack and Pearl Resnick Campus, Bronx, NY, USA, 10461.
}

\section{Abstract}

Caffeic acid (CA) is a hydroxycinnamic acid derivative and polyphenol with antioxidant and antiinflammatory activities. The neuroprotective properties of CA still need detailed characterization in different biological models. Here, the antioxidant and neuroprotective effects of CA were compared in in vitro and in vivo neurotoxic models. Biochemical outcomes of cell dysfunction, oxidative damage and transcriptional regulation were assessed in rat cortical slices, whereas endpoints of physiological stress and motor alterations were characterized in Caenorhabditis elegans (C. elegans). In rat cortical slices, CA $(100 \mu \mathrm{M})$ prevented, in a differential manner, the

*Corresponding author: Abel Santamaría, Ph.D. Laboratorio de Aminoácidos Excitadores, Instituto Nacional de Neurología y Neurocirugía. Insurgentes Sur 3877, Ciudad de México 14269, México. Tel.: (+5255)5606-3822 (x2013); absada@yahoo.com. Conflict of Interest

The authors declare that they have no competing interests, and that the research was conducted in the absence of any commercial or financial relationships that could be construed as a potential conflict of interest. 
loss of reductive capacity, the cell damage, and the oxidative damage induced by the excitotoxin quinolinic acid (QUIN, $100 \mu \mathrm{M})$, the pro-oxidant ferrous sulfate $\left(\mathrm{FeSO}_{4}, 25 \mu \mathrm{M}\right)$, and the dopaminergic toxin 6-hydroxydopamine (6-OHDA, $100 \mu \mathrm{M})$. CA also restored the levels of nuclear factor erythroid 2-related factor 2/Antioxidant Response Element (Nrf2/ARE; a master antioxidant regulatory pathway) binding activity affected by the three toxins. In wild-type (N2) of C. elegans, but not in the skn-1 KO mutant strain (worms lacking the orthologue of mammalian $\mathrm{Nrf2}), \mathrm{CA}(25 \mathrm{mM})$ attenuated the loss of survival induced by QUIN (100 mM), $\mathrm{FeSO}_{4}(15 \mathrm{mM})$ and 6-OHDA ( $25 \mathrm{mM})$. Motor alterations induced by the three toxic models in $\mathrm{N} 2$ and $s k n-1 \mathrm{KO}$ strains were prevented by CA in a differential manner. Our results suggest that: (1) CA affords partial protection against different toxic insults in mammalian brain tissue and in C. elegans specimens; (2) the Nrf2/ARE binding activity participates in the protective mechanisms evoked by CA in the mammalian cortical tissue; (3) the presence of the orthologous skn-1 pathway is required in the worms for CA to exert protective effects; and (4) CA exerts antioxidant and neuroprotective effects through homologous mechanisms in different species.

\section{Keywords}

Antioxidant defense; Neuroprotection; Caffeic acid; Mammal CNS; Caenorhabditis elegans; Transcriptional regulation; Nrf2 pathway; $s k n-1$ pathway

\section{Introduction}

Caffeic acid (3,4-dihydroxycinnamic acid, or CA) is a hydroxycinnamic acid (ColínGonzález et al. 2015) and a catechol secondary product, isolated from plants such as Melissa officinalis $(39.3 \mathrm{mg} / 100 \mathrm{~g}$ ) or Ilex paraguariensis $(15 \mathrm{mg} / 100 \mathrm{~g}$ ) (Khan et al. 2016). CA possesses protective properties such as anticancer, antioxidant and anti-inflammatory activities (Colín-González et al. 2015). The stability of the CA structure increases when hydrogen bonds are generated after breaking $\mathrm{O}-\mathrm{H}$ bonds. The CA antioxidant activity also involves the formation of o-quinone and the regeneration of the $\mathrm{CA}$ structure through semiquinone radical catalyzed by the reaction of the antioxidant with free radicals (Khan et al. 2016). Nonetheless, CA requires validation as a neuroprotective agent with antioxidant properties in neurotoxic models driven by diverse toxic mechanisms, as well as in different species.

Among several neurotoxic models, 6-hydroxydopamine (6-OHDA) accumulates in dopaminergic neurons during toxic episodes, leading to increased generation of reactive oxygen species (ROS) and mitochondrial dysfunction (Shashikumar et al. 2015), whereas quinolinic acid (QUIN) is a competitive agonist for glutamate-sensitive $N$-methyl methyl-Daspartate receptors (NMDAR), leading to excitotoxicity, ROS production and mitochondrial energy depletion (Kotlar et al. 2018). In turn, ferrous sulfate $\left(\mathrm{FeSO}_{4}\right)$ is a pro-oxidant molecule which easily generates hydroxyl radicals $\left(\mathrm{OH}^{*}\right)$ through the Fenton reaction (Lai et al. 2016). Altogether, these toxic models, applied to different biological preparations, serve to establish specific mechanistic features of putative neuroprotective molecules. Moreover, we chose to examine whether treatment with these toxic molecules can be mitigated by CA not only because of their endogenous nature, but also because they may potentially be 
involved in human neurological disorders. For instances, 6-OHDA is likely to be an etiological factor in Parkinson's disease (PD) (Jellinger et al, 1995); QUIN is a toxic metabolite from the kynurenine pathway involved in Huntington's disease (HD) and other neurological disorders (Schwarcz et al, 2010); and $\mathrm{FeSO}_{4}$ is a precursor of $\mathrm{Fe}^{2+}$, a metal ion involved in ROS formation via Fenton reaction once accumulated in the brain (Carocci et al, 2018). Therefore, combined, these toxic models represent valuable experimental approaches to pathological conditions observed in human neurological disorders.

The nematode Caenorhabditis elegans (C. elegans) is a simple and low-cost model due to its small size, transparency, short life cycle, variety of transgenic strains and low cost of maintenance (Shashikumar et al. 2015). It also possesses a small neuronal network (McVey et al. 2010) containing only 302 neurons (Harrington 2010). C. elegans expresses the SKN-1 antioxidant pathway, which is homologous to the well-known Nrf2/ARE antioxidant pathway in mammals (Blackwell et al. 2015; Kotlar et al. 2018; Cuadrado et al. 2019). SKN-1 pathway is key for the worm defense as it confers resistance to oxidative stress by regulating a number of antioxidant enzymes (An and Blackwell 2003; Martinez-Finley et al. 2013). Animals with SKN-1 deletions or modifications are prone to be more sensitive to oxidative stress and have shorter lifespans (An and Blackwell 2003; Kotlar et al. 2018). Combined, these features make $C$. elegans a suitable alternative and complementary model for the characterization of antioxidant and neuroprotective properties of molecules such as CA. Moreover, in order to make inferences related with the mammal CNS derived from the observations obtained from $C$. elegans, it is necessary to compare the effects of antioxidant molecules in worms with mammals. Therefore, aiming to better understand the antioxidant mechanisms underlying the neuroprotective properties of CA, in this study, we compared the capacity of this cinnamic compound to attenuate and/or avoid the deleterious actions of three different neurotoxic models in rat brain cortical slices vs. the nematode $C$. elegans, and whether the protective profile of CA involves the role of Nrf2 in nerve tissue of mammals, and/or its orthologue SKN-1 in the worm.

\section{Materials and methods}

\section{Reagents}

$\mathrm{FeSO}_{4}, 6$-OHDA, QUIN, CA ( $₫ 98.0 \%$ ), thiobarbituric acid (TBA), HEPES, 1323-(4,5dimethylthiazol-2-yl)2,5-diphenyltetrazolium bromide (MTT) and other reagents were obtained from (Sigma-Aldrich, St. Louis, MO). Other reagents were acquired from different commercial sources.

\section{Animals}

C. elegans N2 wild-type and mutant VC1772 (skn-1(ok2315) strains, were purchased from the Caenorhabditis Genetics Center (CGC, University of Minnesota, Minneapolis, MN). Escherichia coli OP50 and NA22 strains were obtained from Laboratorio de Bacteriología (Faculty of Medicine, UNAM, Mexico). Ten male Wistar adult rats (260-280 g) were obtained from the vivarium of the Instituto Nacional de Neurología y Neurocirugía (Mexico), and later euthanized by decapitation in order to obtain the brain and isolate the cortical slices. Before euthanized, rats were kept under room conditions previously 
described (Colonnello et al. 2018). All experiments were carried out in compliance with the "Guidelines for the Use of Animals in Neuroscience Research" from the Society of Neuroscience. All protocols were approved by the Ethics Committee for Animal Research of the Instituto Nacional de Neurología y Neurocirugía (Project No. 126/17). Efforts were made to minimize the number of animals used and their suffering during the experiments.

\section{FRAP assay for iron reduction capacity}

Forty (40) $\mu \mathrm{l}$ of distilled water were added to a Costar 96-well plate. Then, ten $\mu \mathrm{l}$ of CA at increasing concentrations $(50,100,250,500$ and $750 \mu \mathrm{M}$; final concentrations), were incubated with $200 \mu \mathrm{l}$ FRAP solution ( $3.1 \mathrm{mg}$ sodium acetate plus $16 \mathrm{ml}$ acetic acid, $40 \mathrm{mM}$ $\mathrm{HCl}, 10 \mathrm{mM}$ 2,4,6-Tri(2-pyridyl)-s-triazine (TPTZ) and $20 \mathrm{mM} \mathrm{FeCl} 3$ ). Optical density was recorded and processed, and the reduction capacity values were calculated according to a method previously described (Colonnello et al., 2018).

\section{In vitro experiments}

Cell viability assay in cortical slices-Wistar adult male rats (260-280 g) were euthanized by decapitation. The frontal cortex from each rat was rapidly isolated from the brain. Cortical slices (250-300 $\mu \mathrm{m}$ thickness)were obtained with a chopper, according to a previous report (Colonnello et al., 2018) and then incubated in Krebs buffer (124 mM NaCl, $5 \mathrm{mM} \mathrm{KCl}, 1.2 \mathrm{mM} \mathrm{CaCl}_{2}, 1.2 \mathrm{mM} \mathrm{MgSO}_{4}, 1.2 \mathrm{mM} \mathrm{KH}_{2} \mathrm{PO}_{4}, 23 \mathrm{mM} \mathrm{NaHCO}_{3}, 3 \mathrm{mM}$ HEPES and $10 \mathrm{mM} \mathrm{D}$-glucose) for thirty minutes at $37^{\circ} \mathrm{C}$ and $\mathrm{CO}_{2} 5 \%$. Four slices per probe were incubated in the presence of $\mathrm{CA}(100 \mu \mathrm{M})$ for 60 minutes under the same conditions mentioned above. Later on, slices were exposed to toxins QUIN $(100 \mu \mathrm{M})$, $\mathrm{FeSO}_{4}(25 \mu \mathrm{M}$ in ascorbic acid) or 6-OHDA (100 $\mu \mathrm{M}$ in ascorbic acid) and incubated for 60 minutes more. Then, fifteen $\mu \mathrm{L}$ of the MTT reagent $(5 \mathrm{mg} / \mathrm{ml})$ were incorporated to the slices for 60 minutes. The treated cortical slices were rinsed with acidic alcohol (isopropanol in $0.4 \mathrm{~N} \mathrm{HCl}$ ) in order to obtain a purple coloration evidencing mitochondrial metabolism. The optical density from each sample was measured in a Cytation Multifunctional Imaging Reader at $570 \mathrm{~nm}$. Results were calculated as the percentage of MTT reduction vs. the control by interpolation of the absorbance of reduced products per mg of protein (Colonnello et al., 2018).

Lipid peroxidation assay in rat cortical slices-Cortical slices exposed to CA and/or the toxic agents were lysed in $60 \mu \mathrm{L}$ of lysis buffer $(150 \mathrm{mM} \mathrm{NaCl}, 0.1 \%$ Triton, $50 \mathrm{mM}$ Tris $\mathrm{HCl}$ (pH 8.0) and $0.25 \mathrm{mM}$ sucrose); each sample was sonicated for ten seconds until it was completely homogenous. In eppendorf tubes, for every slice, $50 \mu \mathrm{L}$ of the homogenate were added with $100 \mu \mathrm{L}$ of TBA reagent $(0.375 \mathrm{~g}$ TBA reagent plus $15 \mathrm{~g}$ trichloroacetic acid plus $2.5 \mathrm{ml} 1 \mathrm{~N} \mathrm{HCl})$ and boiled for 20 minutes at boiling temperature $\left(96^{\circ} \mathrm{C}\right.$ for Mexico City). All samples were then centrifuged at 12,000 rpm for 15 minutes to obtain the supernatants. Absorbance was read at $532 \mathrm{~nm}$, and values of blank were subtracted from all experiments. A standard curve of 1,1,3,3-tetraethoxypropane (TEP; 20-200 $\mu \mathrm{M}$ ) was constructed to calculate, by extrapolation, the approximate content of TMPO present in each sample generated from the lipid oxidation reaction. Total protein content was quantified by Lowry's method (Lowry et al. 1951). Results were expressed as nmol of TBA-reactive substances (TBARS) produced per mg of protein. 
Assessment of cell damage in cortical slices-To visually define cells undergoing damage, a method based on immunofluorescence was used, according to a previous report (Maya-López et al. 2019). Cortical slices already exposed to the different experimental conditions were loaded with propidium iodide (PI, $100 \mu \mathrm{g} / \mathrm{ml}$, Roche) for $10 \mathrm{~min}$. Then, the medium was removed and slices were washed with PBS three times, fixed with paraformaldehyde (Sigma, 1\%/PBS) for $60 \mathrm{~min}$ and mounted using a DAPI-resin for nuclei counter-stain. Tissue slices were observed with a Cytation 3 device (Bioteck), where images were analyzed by the software provided by the supplier (Gen5 v3.02.2). Also, in order to distinguish neuronal cells from other cell types, slices were previously loaded with antiMap-2 rabbit antibody (Sigma [1:250 dilution]) and secondary anti-rabbit antibody Alexa 488 [1:1000 dilution]). Results were presented as images of simple and triple stains (merge) for DAPI, PI and Map-2.

\section{Separation of cytosolic and nuclear fractions in cortical slices-Cortical slices} were stored at $-70^{\circ} \mathrm{C}$. At the time of use, slices were grinded to powder in a frozen mortar, using liquid nitrogen. Once the tissue was pulverized, nuclear fractions were obtained with the NE-PER kit (Thermo Scientific, Rockford, IL), strictly according with the manufacturer's instructions, supplemented with a protease inhibitor (Mini Protease Inhibitor Cocktail, Roche) and stored at $-70^{\circ} \mathrm{C}$ until use.

\section{Nrf2/ARE binding assay}

Nrf2 activity was determined with the TransAm Nrf2 assay (Active Motif, Carlsbad, CA). Nuclear extracts $(10 \mu \mathrm{g})$ were incubated with immobilized oligonucleotide containing the ARE consensus binding site (5'-GTCACAGTGACTCAGCAGAATCTG-3') on 96-well plates. The active form of Nrf2 that bound the oligo was detected using primary anti-Nrf2 antibody after treating with secondary HRP conjugated antibody. As a result of the specific transcription factor activity in nuclear extracts, the chromogen formed was determined using a Synergy multi-modem plate reader (BioTek, Winooski, VT) at $450 \mathrm{~nm}$, and expressed as Nrf2/ARE binding activity.

\section{In vivo experiments}

C. elegans cultures-Nematodes were grown and maintained using standard procedures. $\mathrm{N} 2$ and $s k n-1$ (ok2315) strains were kept at $21^{\circ} \mathrm{C}$ in a Nematode Growth Medium (NGM) agar plate till they reach gravid stage (Colonnello et al. 2018; Kotlar et al. 2018). Afterwards, 40,000 gravid worms were lysed in a $10 \mathrm{M} \mathrm{NaOH} /$ hypochlorite solution to obtain eggs, which were kept on empty NGM plates overnight to hatch at larva 1 (L1) stage. L1s from both strains were then detached from the plates by washing with $0.5 \%$ saline solution for later assays.

\section{Survival assay}

Approximately 2000 worms in L1 stage from $\mathrm{N} 2$ and $s k n-1$ strains per experiment were first exposed to $25 \mathrm{mM} \mathrm{CA}$ for 30 minutes. Then, nematodes were exposed to the following toxic treatments: $100 \mathrm{mM}$ QUIN, $15 \mathrm{mM} \mathrm{FeSO}$ (in ascorbic acid) or $25 \mathrm{mM}$ 6-OHDA (in ascorbic acid), all for $30 \mathrm{~min}$. The doses of the toxins were determined based on previous reports (Colonnello et al. 2018; Kotlar et al. 2018). Worms were cultured in NGM plates 
containing E. coli (OP50). Twenty-four hours after exposure to toxins, three cells with high density and three cells with low density of worms were transferred to slides and counted, and the averages were divided by six, and then multiplied by the total number of cells (64) to obtain an approximate number of worms in the plate, according to a previous report (Kotlar et al. 2018). Six plates collected from each treatment were considered for this test and the procedure was repeated three times in duplicate.

\section{Body bending assay}

The locomotion rate was measured in $\mathrm{N} 2$ and $s k n-1$ worms 48 hours after exposure to treatments. One worm from each treatment was transferred into a bacteria-free NGM plate. Every worm was allowed to acclimate for 60 seconds. The total number of body bends was quantified during 60 seconds in a stereomicroscope. Each body bending was considered as the change of direction of worm's head characterized by the presence of the pharyngeal bulb towards the right side, following criteria from previous reports (Colonnello et al., 2018; Kotlar et al., 2018). Twelve worms from each group were tested. Tests were repeated six times in duplicate.

\section{Statistical analysis}

All experiments were carried minimally in triplicate. Mean \pm standard error medium (S.E.M.) were determined for all parameters. Statistical significance among groups was determined by two-way analysis of variance followed by Tukey's post-hoc analysis. Values of $P \unlhd 0.05$ were considered as statistically significant.

\section{Results}

\section{CA reduced iron in a concentration-dependent manner}

First, we determined whether CA exerts antioxidant properties per se in an iron reduction assay. Figure 1 shows the iron reduction capacity evoked by increased concentrations of CA $(50-750 \mu \mathrm{M})$. Results demonstrate a concentration-response effect of CA as part of its antioxidant potential, where the $50 \mu \mathrm{M}$ concentration showed the lowest antioxidant capacity (668 $\pm 63 \mu \mathrm{M}$ of iron reduced), whereas the concentration with the most effective reductive capacity was $750 \mu \mathrm{M}(4,230 \pm 232 \mu \mathrm{M}$ of iron reduced). The 100,250 and $500 \mu \mathrm{M}$ concentrations produced a gradual reduction capacity equivalent to $897 \pm 86,1,644 \pm 83$ and $3,003 \pm 189 \mu \mathrm{M}$ or iron reduced, respectively.

\section{CA attenuated the loss of cell viability induced by QUIN and $\mathrm{FeSO}_{4}$ in cortical slices}

The effects of CA on the QUIN (a)-, $\mathrm{FeSO}_{4}$ (b)- and 6-OHDA (c)-induced loss of mitochondrial reductive capacity (as an index of cell viability) are shown in Figure 2. In Figure 2a, QUIN decreased the reductive capacity by $81 \%$ compared with the control $(P \unlhd 0.05)$, whereas preconditioning cortical tissue with CA protected against QUIN inducedtoxicity (152\% above QUIN; $P \unlhd) .05$, different from QUIN; $P \unlhd) .05$, different from the control). In Figure 2b, slices treated only with $\mathrm{FeSO}_{4}$ showed a decrease in reductive capacity (83\%) compared with the control $(P \unlhd) .05)$, whereas pretreatment with CA diminished the toxic effect of $\mathrm{FeSO}_{4}\left(147 \%\right.$ above $\left.\mathrm{FeSO}_{4} ; P \unlhd\right) .05$, different from $\mathrm{FeSO}_{4}$; $P \unlhd 0.05$, different from the control). In Figure 2c, 6-OHDA decreased cell viability by $58 \%$ 
$(P \unlhd 0.05)$ compared with the control, whereas CA did not protect the cortical tissue from the toxic effect of 6-OHDA (4\% below 6-OHDA; $P \unlhd$ 0.05, different from the control). CA per se did not alter cell viability in a significant manner.

\section{CA prevented the oxidative damage to lipids QUIN and 6-OHDA in cortical slices}

Figure 3 depicts the effects of CA on the QUIN (a)-, $\mathrm{FeSO}_{4}$ (b) and 6-OHDA (c)-induced lipid peroxidation (as an index of oxidative damage) in cortical slices. In Figure 3a, QUIN increased the amount of peroxidized lipids (161\%) compared with the control $(P \unlhd) .05)$, whereas CA inhibited this effect (63\% below QUIN; $P \unlhd 0.05$, different from QUIN). In Figure $3 b, \mathrm{FeSO}_{4}$ increased lipid peroxidation (177\%) compared with the control $(P \unlhd 0.05)$; however, $\mathrm{CA}$ was unable to reduce the oxidative damage induced by $\mathrm{FeSO}_{4}(4 \%$ below $\mathrm{FeSO}_{4} ; P \unlhd 0.05$, different from the control). In Figure 3c, 6-OHDA increased the oxidative damage to lipids (92\%) compared with the control $(P<0.05)$, whereas CA protected against 6-OHDA induced-toxicity (51\% below 6-OHDA; $P \unlhd) .05$, different from 6-OHDA). CA per se did not alter lipid peroxidation in a significant manner.

\section{CA reduced the cell damage induced by QUIN and FeSO4 in cortical slices}

The effects of CA on the QUIN-, 6-OHDA- or $\mathrm{FeSO}_{4}$-induced cell damage are shown as a series of fluorescence micrographs in Figure 4. Slices not exposed to toxins or CA (control condition, first line) show low PI staining and prominent positive staining for Map2, meaning that cell permeability is not compromised in this condition, and a large number of neuronal cells can be found, as evidenced also in the merge column. In the second line, CA per se did not induce changes in PI or Map2 stains compared with the control (3\% above the control value). In contrast to the control condition, slices exposed to QUIN, 6-OHDA or $\mathrm{FeSO}_{4}$ (third, fourth and fifth lines, respectively) displayed strong PI stains (180\%, 121\% and $126 \%$ above the control, respectively; $P \unlhd 0.05$ ) and lowered responses to Map2. Slices incubated in the presence of $\mathrm{CA}+\mathrm{QUIN}$ (sixth line) and $\mathrm{CA}+\mathrm{FeSO}_{4}$ (eight line) showed moderate increase in the PI staining compared with the control, but these fluorescent signals were lower than those of their respective toxic controls (57\%, and 45\%, respectively; $P \unlhd$ 0.05). In addition, in all toxic conditions challenged with CA, Map2 labelling was preserved to similar extent as in control conditions. The level of staining for PI remained similar in the 6-OHDA (seventh line) and CA+6-OHDA conditions in the images, although the densitometric analysis revealed that CA readily (but moderately) decreased PI incorporation (39\% below CA+6-OHDA; $P \unlhd$ D.05). Merged images show several PI positive nuclei matching with DAPI positive nuclei in the micrographs corresponding to the toxic conditions (last column; third, fourth and fifth lines).

\section{CA preserved the Nrf2/ARE binding activity decreased by QUIN, $\mathrm{FeSO}_{4}$ and 6-OHDA in cortical slices}

The effects of CA on the QUIN (a)-, $\mathrm{FeSO}_{4}$ (b)- and 6-OHDA (c)-induced decrease in Nrf2/ARE axis activation are shown in Figure 5. In Figure 5a, QUIN decreased this endpoint by $17 \%$ compared with the control $(P \unlhd) .05)$, whereas CA protected against QUIN inducedtoxicity (20\% above QUIN; $P \unlhd$ 0.05, different from QUIN). As shown in Figure 5b, slices treated with $\mathrm{FeSO}_{4}$ showed a decrease of Nrf2/ARE binding activity (27\%) compared with 
the control $(P \unlhd 0.05)$, whereas pretreatment with CA diminished the toxic effect of $\mathrm{FeSO}_{4}$ (45\% above $\mathrm{FeSO}_{4} ; P \unlhd$ 0.05, different from $\mathrm{FeSO}_{4}$ ). As shown in Figure 5c, 6-OHDA decreased Nrf2/ARE activation by $20 \%(P \unlhd 0.05)$ compared with the control, whereas CA protected the cortical tissue from the toxic effect of 6-OHDA (25\% above 6-OHDA; $P \unlhd) .05$, different from 6-OHDA). CA per se did not alter this marker.

\section{CA increased the survival of $\mathrm{N} 2$ (wild-type) strain of $C$. elegans exposed to QUIN, $\mathrm{FeSO}_{4}$ or 6-OHDA, but it did not incrased survival in the skn-1 KO strain}

Survival of N2 and mutant $s k n-1$ (ok2315) strains was tested 24 hours after exposure to toxic treatments (Figures 6a-6f). For both worm strains, the percentage of survival in the control groups was considered as $100 \%$. As shown in Figures 6a-6c, QUIN, $\mathrm{FeSO}_{4}$ and 6-OHDA decreased the survival rate in $\mathrm{N} 2$ worms by $49 \%, 42 \%$ and $38 \%$ below the control, respectively ( $P \unlhd 0.05$, different from the control for all cases), whereas in the presence of $\mathrm{CA}$, survival levels remained significant from control, but nevertheless significantly attenuated to death/survival upon treatments (20\% above QUIN [P $P \unlhd .05$, different from the control; $P \unlhd 0.05$, different from QUIN], $17 \%$ above $\mathrm{FeSO}_{4}[P \unlhd 0.05$, different from the control; $P \unlhd 0.05$, different from $\left.\mathrm{FeSO}_{4}\right]$, and $18 \%$ above 6-OHDA [ $P \unlhd 3.05$, different from the control; $P \unlhd$.05, different from 6-OHDA).

In the $s k n-1$ (ok2315) strain (Figures 6d-6f), the survival rate was diminished by QUIN $(28 \%), \mathrm{FeSO}_{4}(41 \%)$ and 6-OHDA (37\%) compared with the control $(P \unlhd) .05$, different from the control for all cases). In contrast to its effects on N2 strain, CA did not exert any effect on the survival rate in $s k n-1 \mathrm{KO}$ strain upon QUIN, $\mathrm{FeSO}_{4}$ or 6-OHDA treatments (28\%, $38 \%$ and $28 \%$ below the control, respectively; $P \leq 0.05$, different from the control for all cases). These results clearly suggest that the presence of the SKN-1 pathway is required for CA to exert protective and antioxidant effects as it was previously described by Colonnello et al. (2018).

\section{CA protected N2 and skn-1 KO worms against motor alterations induced by QUIN, $\mathrm{FeSO}_{4}$ and 6-OHDA in a differential manner}

Locomotion rate was quantified 48 hours after exposure of N2 and $s k n-1$ (ok2315) strains to toxic treatments (Figure 7). The average number of body bends per worm in the control N2 group was $35 \pm 1$ (body bending/1 minute). N2 worms (Figures 7a-7c) exposed to QUIN, $\mathrm{FeSO}_{4}$ and 6-OHDA presented either hyperactive or hypoactive motor alterations, expressed as an altered number of body bends ( $40 \pm 1,29 \pm 1$ and $28 \pm 2$, respectively; $P \leq 0.05$, different from the control for all cases). CA ameliorated motor disturbances induced by QUIN ( $34 \pm 0.8 ; P \unlhd 0.05$, different from QUIN), $\mathrm{FeSO}_{4}(35 \pm 1 ; P \unlhd 0.05$, different from $\left.\mathrm{FeSO}_{4}\right)$ and 6-OHDA (34 $\pm 2 ; P \unlhd 0.05$, different from 6-OHDA).

The average number of body bends per worm in the $s k n-1 \mathrm{KO}$ control group was $29 \pm 0.8$ (body bending/1 minute). Mutant skn-1(ok2315) worms (Figures 7d-7f) exposed to QUIN, $\mathrm{FeSO}_{4}$ and 6-OHDA showed hypoactive motor behaviors (18 $\pm 0.6,25 \pm 1$ and $20 \pm 0.6$, respectively; $P \leq 0.05$, different from the control for all cases). CA exerted ameliorative effects on behavioral alterations in worms exposed to QUIN ( $28 \pm 1 ; P \unlhd) .05$, different from QUIN) and 6-OHDA (28 $\pm 1 ; P \unlhd) .05$, different from 6-OHDA). Worms preconditioned with 
$\mathrm{CA}$ and exposed to $\mathrm{FeSO}_{4}$ did not show any recovery in this behavioral endpoint $(25 \pm 0.7$; $P \unlhd$.05, different from the control).

\section{Discussion}

In this study, we demonstrate that CA, likely by means of its antioxidant properties (confirmed in Figure 1 as its reductive capacity of iron), attenuates the loss of cell viability induced by the excitotoxic molecule QUIN and the pro-oxidant $\mathrm{FeSO}_{4}$, but not the effect of the parkinsonian toxin 6-OHDA (Figure 2). CA also prevented the oxidative damage to lipids induced by QUIN and 6-OHDA, but not the effect of $\mathrm{FeSO}_{4}$ (Figure 3). Neuronal cell damage was reduced by CA only for QUIN and $\mathrm{FeSO}_{4}$, but only moderately for 6-OHDA (Figure 4). The differential protective effects exerted by CA on all these selective toxic models also seem to be subordinated to the master regulator of antioxidant responses, the Nrf2/ARE pathway, as suggested by the results shown in Figure 5. In addition, in vivo experiments revealed that $\mathrm{CA}$ is effective in preventing the reduction of survival in wild type N2 C. elegans, but not in skn-1 KO worms (Figure 6), therefore suggesting an active role of the SKN-1 pathway in the protective efficacy afforded by CA. It is noteworthy, CA protected wild type worms against motor alterations induced by all toxins, but failed to protect $\mathrm{FeSO}_{4}$ exposed specimens (Figure 7), suggesting a complex regulation of the phenolic compound on motor activity in vivo.

This is, to our knowledge, the first report compiling comparative evidence on the protective properties of CA in different biological preparations exposed to toxins with different mechanistic profiles, and involving the activation of orthologous transcriptional regulatory systems. Most of the protective effects of CA have been attributed to derived and/or related compounds, such CA phenethyl ester; CA per se has been shown to evoke antioxidant and neuroprotective effects. We have recently published experimental evidence showing that CA exerted neuroprotective properties against excitotoxic damage induced by QUIN in the rat striatum at the behavioral and biochemical levels (Colín-González et al. 2015). It is assumed, from our current findings about the reductive capacity and the antiperoxidative effects of CA in the toxic models tested, as well as from other previous reports (Kalonia et al. 2009; Sul et al. 2009; Kumar et al. 2010; Jeong et al. 2011; Szwajgier et al. 2017), that the protective effects evoked by CA are due to the antioxidant properties already reported for this phenolic compound, which in turn might strongly account for its neuroprotective profile. Consequently, the neuroprotection afforded by $\mathrm{CA}$ in this and other reports via the stimulation of antioxidant activity, is likely to be responsible for improvement and recovery of diverse physiological functions in toxic models, including the re-establishment of the mitochondrial reductive capacity in cortical cells accompanied by the early activation of transcriptional pathways, and the preservation of survival and motor performance in worms all observed herein-, as well as the direct scavenging of intracellular ROS (Jeong et al. 2011), the regulation of calcium influx to cells (Sul et al. 2009), the recovery of the striatal glutathione redox status, and the prevention of altered locomotor activity in experimental animal models (Kalonia et al. 2009).

Although CA exerted protective effects on most of the endpoints evaluated and the toxic models tested herein in an effect attributable to its antioxidant capacity, we found that this 
phenolic exerted some selective effects, showing poor or no efficacy in several of the tested outcomes. Though CA prevented the 6-OHDA-induced lipid peroxidation and the decrease in Nrf2/ARE binding activity, it did not improve the loss of cell viability induced by 6OHDA. These findings indicate that even though CA can stimulate antioxidant activity in this toxic model, this strategy is insufficient to protect cells completely from the damage induced by 6-OHDA, probably due to the involvement of other mechanisms in its toxicity. In this regard, and as additional corroboration for these considerations, we were able to find only one report mooting the use of CA in the 6-OHDA toxic model (Gunjima et al. 2014). These authors compared the effects of 3,4-dihydroxybenzalacetone (DBL) and CA in SY5Y cells exposed to 6-OHDA, and found that only DBL improved cell survival in this model while promoted Nrf2 nuclear translocation. It is therefore, not surprising to find no reports about this topic, as CA seems to be ineffective in affording protection in rat cortical slices subjected to this toxin, whatever the mechanisms involved. However, it remains noteworthy that CA was protective in wild type $C$. elegans specimens exposed to 6-OHDA, suggesting that, for this biological model, antioxidant and neuroprotective strategies act in a coordinated and effective manner to reduce 6-OHDA toxicity when stimulated with CA, also probably implying that the toxic mechanisms exerted by 6-OHDA are not precisely the same (in nature and/or intensity) in mammals and nematodes.

In contrast to the above described effects, CA was consistently effective in preventing QUIN- and $\mathrm{FeSO}_{4}$-induced loss of cell viability, cell damage and Nrf2/Are binding activity, suggesting that the phenolic compound, by means of early transcriptional regulation mechanisms, counteracted excitotoxicity and oxidative damage evoked by these two toxic models. For the case of QUIN, this concept is also reinforced by the efficacy of CA to reduce lipid peroxidation; however, the phenolic compound did not change the lipoperoxidative action of $\mathrm{FeSO}_{4}$. Accordingly, we hypothesize that the effect of $\mathrm{FeSO}_{4}$-a strong pro-oxidant agent directly producing ROS- was so intense on lipids oxidation that $\mathrm{CA}$, at the concentration tested, was unable to prevent oxidative damage. Moreover, it is likely that, in this study, $\mathrm{FeSO}_{4}$ exerted a stronger pro-oxidant and neurotoxic activity than in a previous report of our group (Colonnello et al. 2018), as judging by its effect on the locomotion of $\mathrm{N} 2 \mathrm{C}$. elegans strain; while in that report $\mathrm{FeSO}_{4}$ induced hyperactivity, here this agent led worms to hypoactive motor behavior, which can be assumed as a more damaging behavioral expression. The simple explanation of this discrepancy could be explained by a technical issue: in this study, iron was prepared in ascorbic acid, thus preserving its pro-oxidant properties and potency for a longer time. Nonetheless, further studies should be directed at other endpoints in cortical slices, such as protein oxidation or glutathione levels, which might shed further light on the antioxidant effect of CA in the $\mathrm{FeSO}_{4}$ toxic model. In addition, these results are in agreement with a previous report demonstrating protective effects of $\mathrm{CA}$ on QUIN- and $\mathrm{FeSO}_{4}$-induced toxicity in rat brain tissue, evidenced by the inhibition of acetylcholinesterase (AChE) and butyrylcholinesterase $(\mathrm{BChE})$ activation and reduction of oxidative damage (Oboh et al. 2013).

It is noteworthy, CA was effective in improving survival and motor performance in wild type $\mathrm{N} 2$ C. elegans exposed to QUIN, $\mathrm{FeSO}_{4}$ and 6-OHDA, supporting the concept that redox regulation in the worms is crucial for survival and other main aspects of the animals' physiology. Meanwhile, CA protection on survival was eliminated in $s k n-1$ mutants (Figures 
6a-6c), indicating $\mathrm{CA}$ is able to activate $\mathrm{SKN}-1$ signaling. However, the situation was more complicated in the nervous system. We noticed that CA protected the locomotion defect induced by QUIN and 6-OHDA in skn-1 KO worms (Figures 7d-7f), which suggests CA might act through additional signaling pathways other than SKN-1 in the nervous system. Pietsch et al. (2011) demonstrated that among the CA properties involved in hormesis, life extension and thermotolerance improved in $C$. elegans, its antioxidative capacity and the regulation of osr-1, sek-1, sir-2.1 and unc-43 plus daf-16 genes are in the first line of relevance. Therefore, it is likely that $\mathrm{CA}$ activates $d a f-16$ and/or the above mentioned genes in the $C$. elegans nervous system, in addition to the SKN-1 signaling pathway. Furthermore, the selective activation of substrate signaling pathways by $\mathrm{CA}$ may be dependent on different toxins, when comparing the protection of $\mathrm{CA}$ against $\mathrm{FeSO}_{4}$, QUIN or 6-OHDA (Figures $7 \mathrm{~d}-7 \mathrm{f}$ ). Further research is required to investigate additional signaling pathways regulated by $\mathrm{CA}$ in the nervous system.

The selective protective effects of CA observed in this study in cortical slices are in contrast with the broad profile of neuroprotective actions recently reported for this phenolic compound in primary cultures of rat cerebellar granule neurons (Taram et al. 2016). The authors demonstrate that CA, by means of its antioxidant capacity, was able to reduce nitric oxide-induced nitrosative stress, glutamate-induced excitotoxicity, hydrogen peroxide toxicity, proteasome inhibition, caspase-dependent intrinsic apoptosis, and endoplasmic reticulum stress. Though there is no a simple explanation for this discrepancy, in an attempt to explain why CA can display such a broad protective spectrum in primary neuronal cell cultures, but a more selective effect in slices, it is necessary to consider that cell cultures represent a single response limited to a single type of cells, whereas tissue slices comprise a complex response of multiple cells.

The protective effects of $\mathrm{CA}$ and its derivatives have been investigated in various model systems, including in vivo. Although the current study adds new insights into the understanding of the beneficial effects of CA by demonstrating the involvement of Nrf2/ARE and SKN-1 pathways in their respective models, the mechanisms underlying the role of these pathways remain unclear. In this regard, polyphenols, such as chlorogenic acid (Yao et al. 2019), honokiol (Hou et al. 2018) and hydroxytyrosol (Peng et al. 2015), are well known to have the ability to activate Nrf2 via the redox cycling intermediate ortho- or paraquinone. A similar mechanism triggered by CA could take place as this polyphenol induces the formation of a caffeic acid ortho-quinone, with further cycling reactions involved (Weber et al. 2019). In turn, the involvement of different signaling pathways, such as the MEK/ ERK1/2 and PI3K/Akt pathways, in the neuroprotective effects of CA has been previously reported (Moosavi et al. 2015), and could eventually recruit Nrf2 activation. Whether these mechanisms are occurring in the models tested in the current study, remain to be elucidated. In the meantime, herein, the CA-activated Nrf2 pathway, and the requirement for the expression of $s k n-1$ for the protective mechanisms exerted by the phenolic compound, appear analogous in both mammals and nematodes; therefore, orthologous defense pathways may act through homologous mechanisms to coordinate antioxidant and neuroprotective responses. On this basis, the $C$. elegans model constitutes itself as a valuable research tool to assess toxic and protective protocols currently tested in mammalian tissue, likely providing similar inferences. 
In summary, the neuroprotective and antioxidant effects evoked by CA in the tested models of this study seem to be mostly subordinated to its direct antioxidant/scavenging properties, but also to its capacity to recruit the orthologous Nrf2 (for mammals) and $s k n-1$ (for $C$. elegans) transcriptional antioxidant pathways. Therefore, CA constitutes another viable example of a naturally occurring antioxidant recruiting direct and/or regulated mechanisms to recover normoxia and homeostasis in the CNS. This is relevant as it establishes solid basis for the design of therapeutic strategies against neurodegenerative events based on molecules exhibiting multifaceted properties.

\section{Conclusion}

In this study, CA induced protection against the different toxic insults tested in rat cortical tissue and in $C$. elegans specimens in a partial-dependent manner. The differences observed in the patterns of protection evoked by CA throughout the toxic models tested suggest specific properties of the antioxidant to counteract selective toxic mechanisms and features in both biological preparations. It is also evident that Nrf2 and $s k n-1$ pathways participate in the protective mechanisms exerted by $\mathrm{CA}$ in the mammalian cortical tissue and in $\mathrm{C}$. elegans, respectively; therefore, our results suggest that CA exerts antioxidant and neuroprotective effects through homologous mechanisms in different species.

\section{Acknowledgements}

The authors wish to express gratitude to María José Aguirre-González, Diana Ruiz-Hernández and Ilan Kotlar for their excellent technical assistance.

Funding Information

This work was supported by CONACYT-TUBITAK grant 265991 (CONACYT/SRE given to AS). MA was supported in part by grants from the National Institute of Environmental Health Sciences, R01ES03771 and R01ES10563.

\section{References}

An JH, Blackwell TK (2003) SKN-1 links C. elegans mesendodermal specification to a conserved oxidative stress response. Genes Dev 17:1882-1893. [PubMed: 12869585]

Blackwell TK, Steinbaugh MJ, Hourihan JM, Ewald CY, Isik M (2015) SKN-1/Nrf, stress responses, and aging in Caenorhabditis elegans. Free Radic Biol Med 88(Pt B):290-301. [PubMed: 26232625]

Carocci A, Catalano A, Sinicropi MS, Genchi G (2018) Oxidative stress and neurodegeneration: the involvement of iron. Biometals 31:715-735. [PubMed: 30014355]

Colín-González AL, Sánchez-Hernández S, de Lima ME, Ali SF, Chavarría A, Villeda J, Santamaría A (2015) Protective effects of caffeic acid on quinolinic acid-induced behavioral and oxidative alterations in rats. J Drug Alcohol Res 4:1-5.

Colonnello A, Kotlar I, de Lima ME, Ortíz-Plata A, García-Contreras R, Soares FAA, Aschner M, Santamaría A (2018) Comparing the effects of ferulic acid and sugarcane aqueous extract in in vitro and in vivo neurotoxic models. Neurotox Res. 34:640-648. [PubMed: 29949107]

Cuadrado A, Rojo AI, Wells G, Hayes JD, Cousin SP, Rumsey WL, Attucks OC, Franklin S, Levonen A-L, Kensler TW, Dinkova-Kostova AT (2019) Therapeutic targeting of the NRF2 and KEAP1 partnership in chronic diseases. Nature Rev Drug Discovery 18:295-317. [PubMed: 30610225]

Gunjima K, Tomiyama R, Takakura K, Yamada T, Hashida K, Nakamura Y, Konishi T, Matsugo S, Hori O (2014) 3,4-dihydroxybenzalacetone protects against Parkinson's disease-related neurotoxin 6-OHDA through Akt/Nrf2/glutathione pathway. J Cell Biochem 115:151-160. [PubMed: 23959789] 
Harrington AJ, Hamamichi S, Caldwell GA, Caldwell KA (2010) C. elegans as a model organism to investigate molecular pathways involved with Parkinson's disease. Dev Dyn 239:1282-1295. [PubMed: 20108318]

Hou Y, Peng S, Li X, Yao J, Xu J, Fang J (2018) Honokiol alleviates oxidative stress-induced neurotoxicity via activation of Nrf2. ACS Chem Neurosci 9:3108-3116. [PubMed: 29989791]

Jellinger K, Linert L, Kienzl E, Herlinger E, Youdim MB (1995) Chemical evidence for 6hydroxydopamine to be an endogenous toxic factor in the pathogenesis of Parkinson's disease. J Neural Transm Suppl 46:297-314. [PubMed: 8821067]

Jeong CH, Jeong HR, Choi GN, Kim DO, Lee U, Heo HJ (2011) Neuroprotective and anti-oxidant effects of caffeic acid isolated from Erigeron annuus leaf. Chin Med 6:25. [PubMed: 21702896]

Kalonia H, Kumar P, Kumar A, Nehru B (2009) Effects of caffeic acid, rofecoxib, and their combination against quinolinic acid induced behavioral alterations and disruption in glutathione redox status. Neurosci Bull 25:343-352. [PubMed: 19927170]

Khan FA, Maalik A, Murtaza G (2016) Inhibitory mechanism against oxidative stress of caffeic acid. J Food Drug Anal 24:695-702. [PubMed: 28911606]

Kotlar I, Colonnello A, Aguilera-González MF, Avila DS, de Lima ME, García-Contreras R, OrtízPlata A, Soares FAA, Aschner M, Santamaría A (2018) Comparison of the toxic effects of quinolinic acid and 3-nitropropionic acid in C. elegans: involvement of the SKN-1 pathway. Neurotox Res 33:259-267. [PubMed: 28822104]

Kumar A, Vashist A, Kumar P (2010) Potential role of pioglitazone, caffeic acid and their combination against fatigue syndrome-induced behavioural, biochemical and mitochondrial alterations in mice. Inflammopharmacology 18:241-251. [PubMed: 20602174]

Lai W, Wei Q, Zhuang J, Lu M, Tang D (2016) Fenton reaction-based colorimetric immunoassay for sensitive detection of brevetoxin B. Biosens Bioelectron 80:249-256. [PubMed: 26851583]

Lowry OH, Rosebrough NJ, Farr AL, Randall RJ (1951) Protein measurement with the Folin-phenol reagent. J Biol Chem 193:265-275. [PubMed: 14907713]

Martinez-Finley EJ, Caito S, Slaughter JC, Aschner M (2013) The role of skn-1 in methylmercuryinduced latent dopaminergic neurodegeneration. Neurochem Res 38:2650-2660. [PubMed: 24194349]

Maya-López M, Rubio-López LC, Rodríguez-Alvarez IV, Orduño-Piceno J, Flores-Valdivia Y, Colonnello A, Rangel-López E, Túnez I, Prospéro-García O, Santamaría A (2019) A cannabinoid receptor-mediated mechanism participates in the neuroprotective effects of oleamide against excitotoxic damage in rat brain synaptosomes and cortical slices. Neurotox Res In press doi: 10.1007/s12640-019-00083-1.

McVey M (2010) Strategies for DNA interstrand crosslink repair: insights from worms, flies, frogs, and slime molds. Environ Mol Mutagen 51:646-658. [PubMed: 20143343]

Moosavi F, Hosseini R, Saso L, Firuzi O (2015) Modulation of neurotrophic signaling pathways by polyphenols. Drug Des Devel Ther 10:23-42.

Oboh G, Agunloye OM, Akinyemi AJ, Ademiluyi AO, Adefegha SA (2013) Comparative study on the inhibitory effect of caffeic and chlorogenic acids on key enzymes linked to Alzheimer's disease and some pro-oxidant induced oxidative stress in rats' brain-in vitro. Neurochem Res 38:413-419. [PubMed: 23184188]

Peng S, Zhang B, Yao J, Duan D, Fang J (2015) Dual protection of hydroxytyrosol, an olive oil polyphenol, against oxidative damage in PC12 cells. Food Funct 6:2091-100. [PubMed: 26037629]

Pietsch K, Saul N, Chakrabarti S, Stürzenbaum SR, Menzel R, Steinberg CE (2011) Hormetins, antioxidants and prooxidants: defining quercetin-, caffeic acid- and rosmarinic acid-mediated life extension in C. elegans. Biogerontology 12:329-347. [PubMed: 21503726]

Schwarcz R, Guidetti P, Sathyasaikumar KV, Muchowski PJ (2010) Of mice, rats and men: Revisiting the quinolinic acid hypothesis of Huntington's disease. Prog Neurobiol 90:230-245. [PubMed: 19394403]

Shashikumar S, Pradeep H, Chinnu S, Rajini PS, Rajanikant GK (2015) Alpha-linolenic acid suppresses dopaminergic neurodegeneration induced by 6-OHDA in $C$. elegans. Physiol Behav 151:563-569. [PubMed: 26300470] 
Sul D, Kim HS, Lee D, Joo SS, Hwang KW, Park SY (2009) Protective effect of caffeic acid against beta-amyloid induced neurotoxicity by the inhibition of calcium influx and tau phosphorylation. Life Sci 84:257-262. [PubMed: 19101570]

Szwajgier D, Borowiec K, Pustelniak K (2017) The neuroprotective effects of phenolic acids: molecular mechanism of action. Nutrients 9: 477.

Taram F, Winter AN, Linseman DA (2016) Neuroprotection comparison of chlorogenic acid and its metabolites against mechanistically distinct cell death-inducing agents in cultured cerebellar granule neurons. Brain Res 1648:69-80. [PubMed: 27444557]

Weber F, Engelke GH, Schieber A (2019) Structure elucidation and tentative formation pathway of a red colored enzymatic oxidation product of caffeic acid. Food Chem 297:124932. [PubMed: 31253253]

Yao J, Peng S, Xu J, Fang J (2019) Reversing ROS-mediated neurotoxicity by chlorogenic acid involves its direct antioxidant activity and activation of Nrf2-ARE signaling pathway. BioFactors 45:616-626. [PubMed: 30951611] 


\section{CA Iron Potential Reduction}

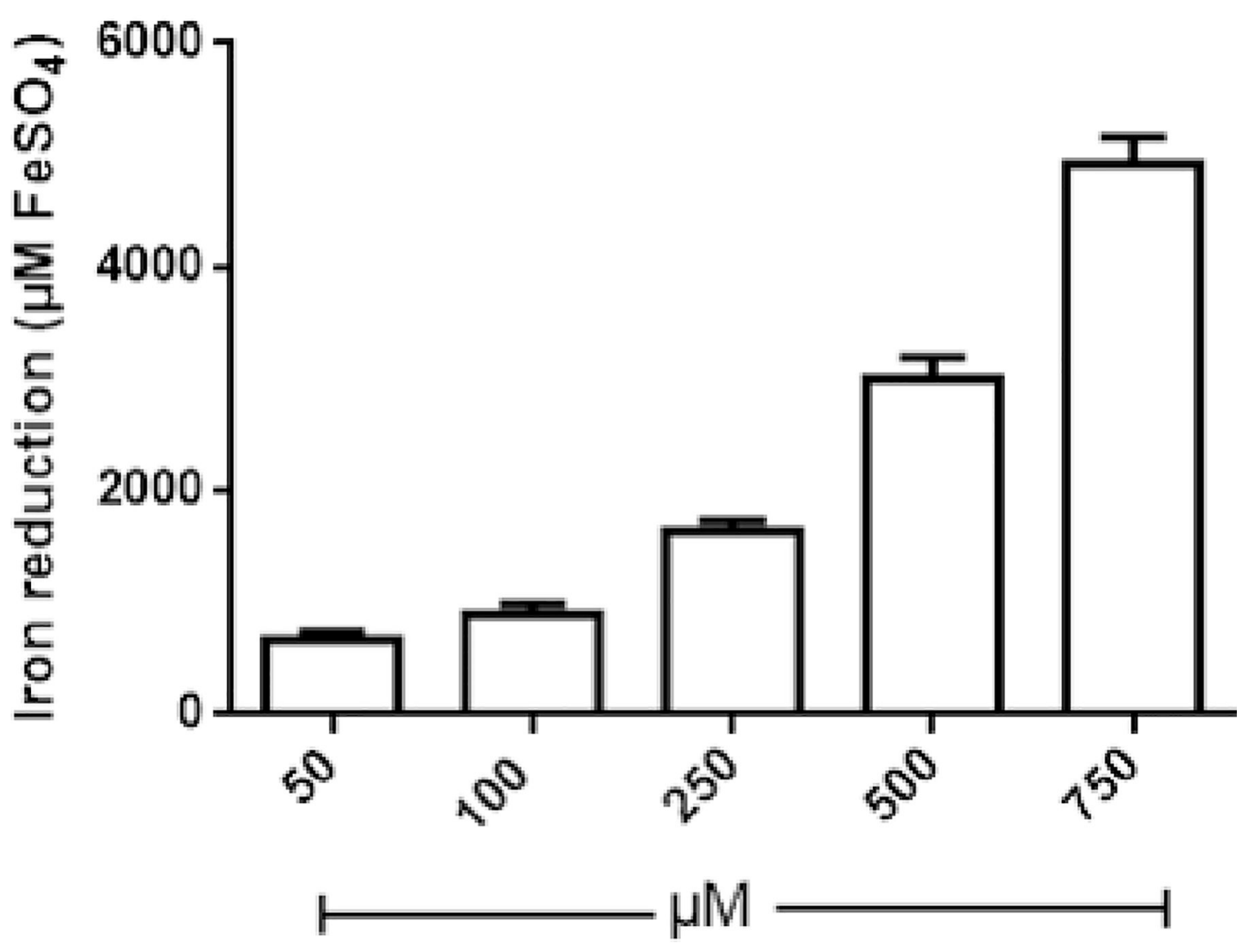

Figure 1.

Iron reduction potential of caffeic acid (CA) in the FRAP assay. Values represent means \pm SEM of $n=12$ experiments per bar. 

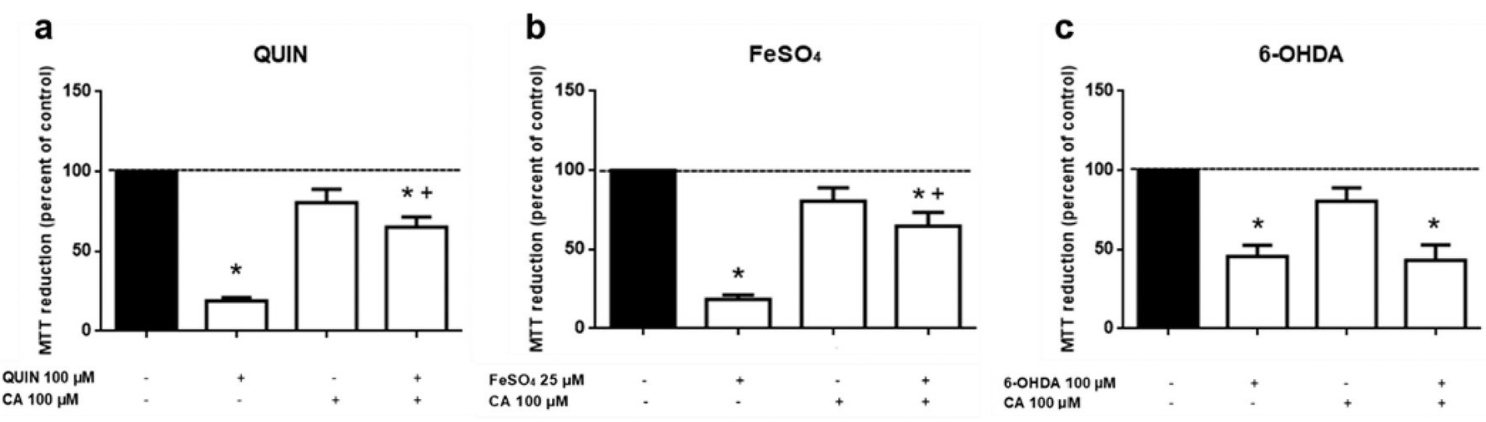

Figure 2.

Effects of caffeic acid (CA) on quinolinic acid (QUIN; a)-, ferrous sulfate $\left(\mathrm{FeSO}_{4}\right.$; $\left.\mathbf{b}\right)$ - or 6hydroxydopamine (6-OHDA; c)-induced loss of cell viability in rat cortical slices. Values represent means \pm S.E.M. ( $\mathrm{n}=6$ experiments per group). $\left.{ }^{*} P \unlhd\right) .05$, different from the control; ${ }^{+} P \unlhd 0.05$, different from the toxin tested; two-way analysis of variance followed by post hoc Tukey's test. 
QUIN

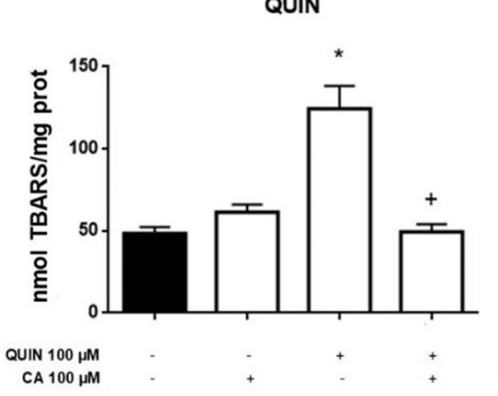

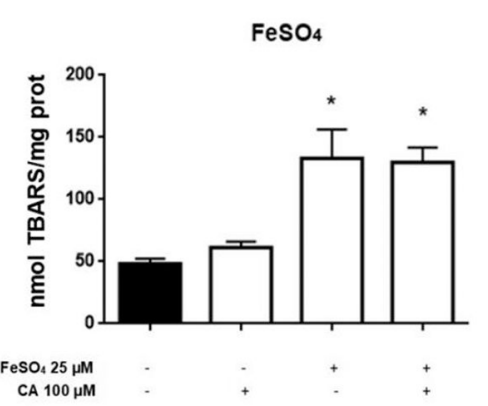

6-OHDA

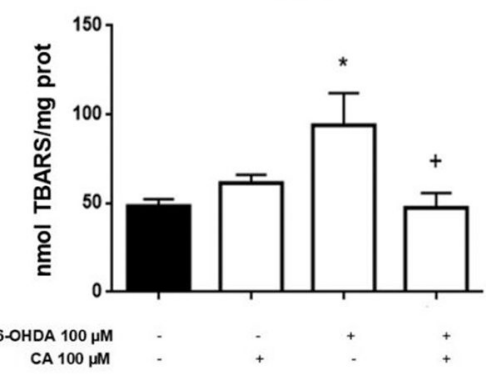

Figure 3.

Effects of caffeic acid (CA) on quinolinic acid (QUIN; a)-, ferrous sulfate $\left(\mathrm{FeSO}_{4}\right.$; b)- or 6hydroxydopamine (6-OHDA; c)-induced lipid peroxidation in rat cortical slices. Values represent means \pm S.E.M. $(\mathrm{n}=5-8$ experiments per group). $* P \unlhd 0.05$, different from the control; ${ }^{+} P \unlhd 0.05$, different from the toxin tested; two-way analysis of variance followed by post hoc Tukey's test. 


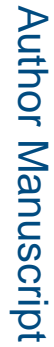

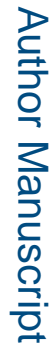

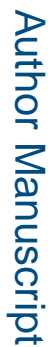

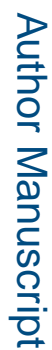

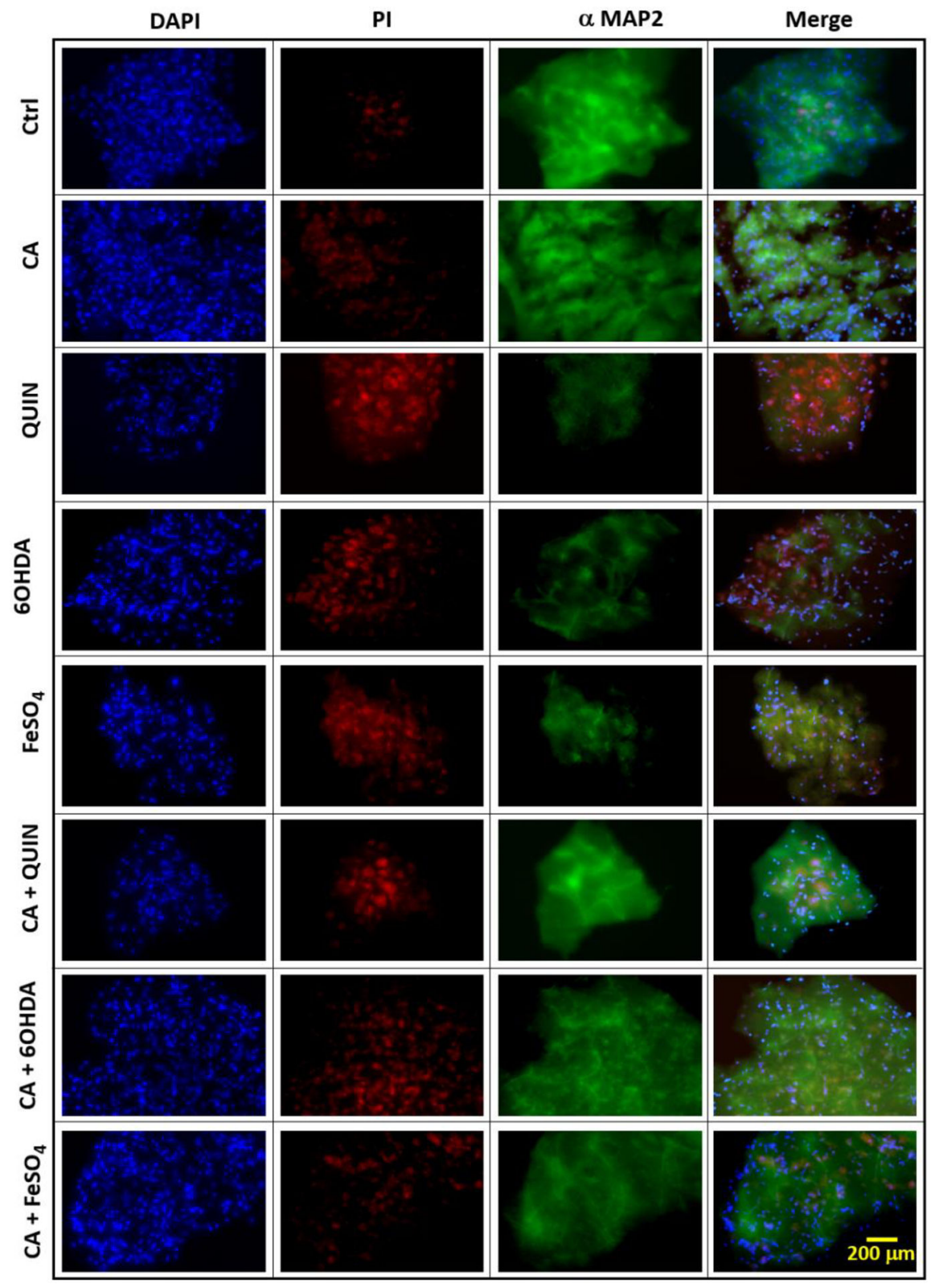




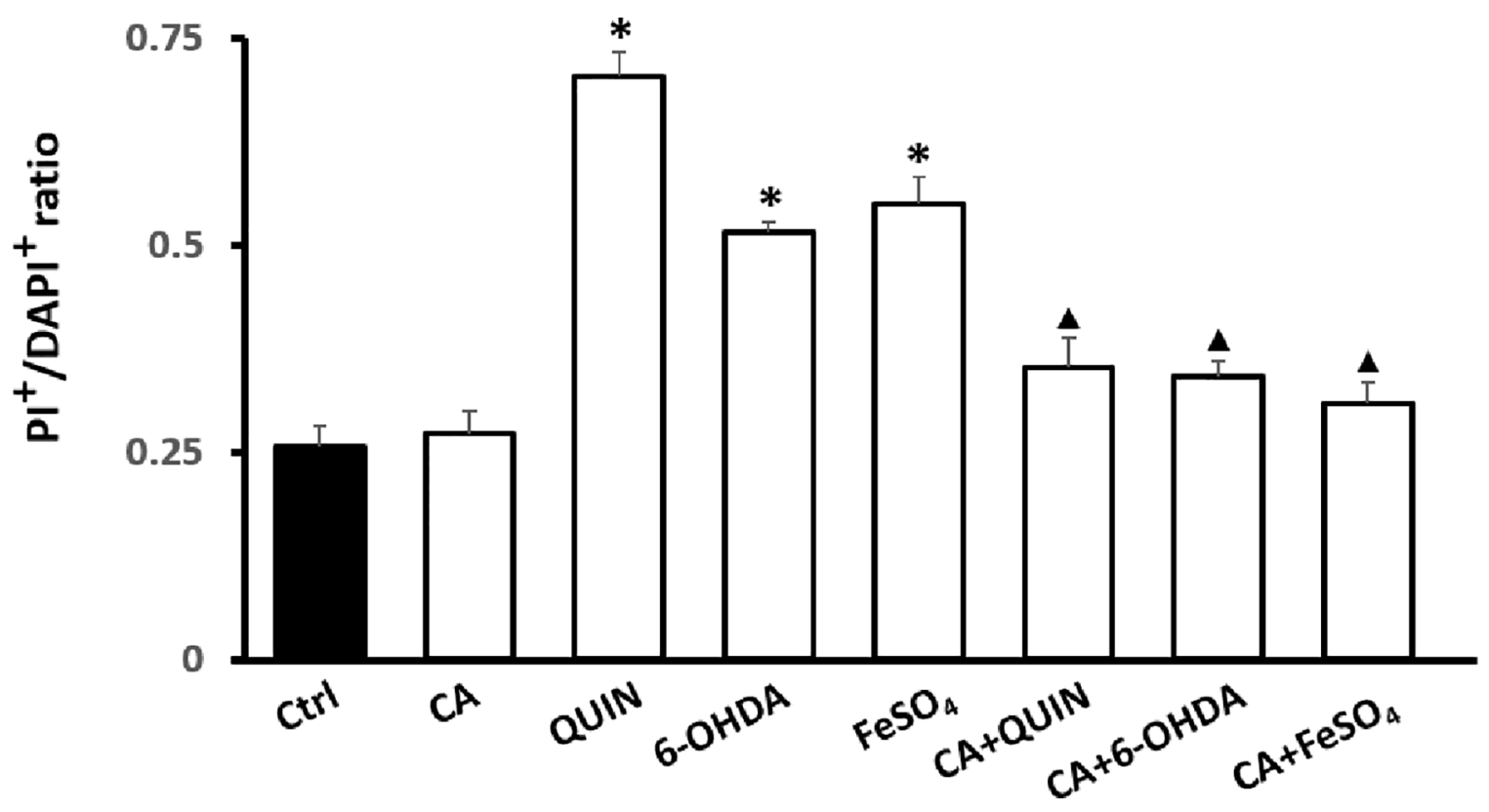

Figure 4.

Effect of caffeic acid (CA) on quinolinic acid (QUIN)-, ferrous sulfate $\left(\mathrm{FeSO}_{4}\right)$ - or 6hydroxydopamine (6-OHDA)-induced cell damage in rat cortical slices. Fluorescence micrographs cell nuclei of slices exposed to QUIN, $\mathrm{FeSO}_{4}$ or 6-OHDA stained with 4',6diamidino-2-phenylindole (DAPI) are shown in the first (left) column. In the second column (middle left), fluorescence micrographs of cells positive to propidium iodide (PI) exposed to the same treatments is depicted. The third column (middle right) shows the fluorescent signal of cortical slices positive to Map2 protein as a marker of neuronal cell labelling. Merge for DAPI + PI + Map2 is depicted in micrographs from the fourth (right) column. Bar line corresponds to $200 \mu \mathrm{m}$ scale. All fields correspond to $40 \mathrm{X}$ magnifications. In the bottom panel, the densitometric analysis shows the PI/DAPI ratio. Values represent means \pm S.E.M. ( $\mathrm{n}=3$ experiments per group). $* P \unlhd 0.05$, different from the control; $\boldsymbol{\Delta} P \unlhd 0.05$, different from the toxin tested; two-way analysis of variance followed by post hoc Tukey's test. 


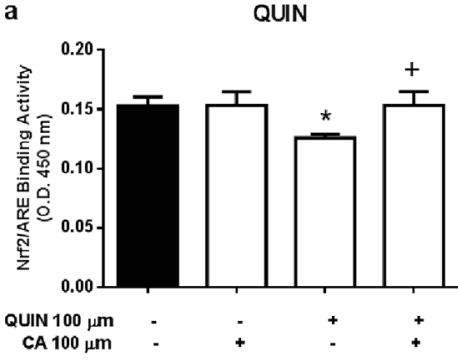

b

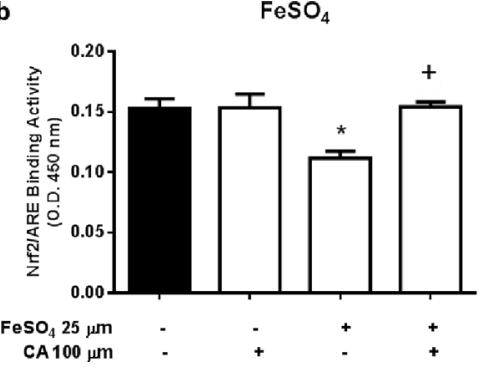

c

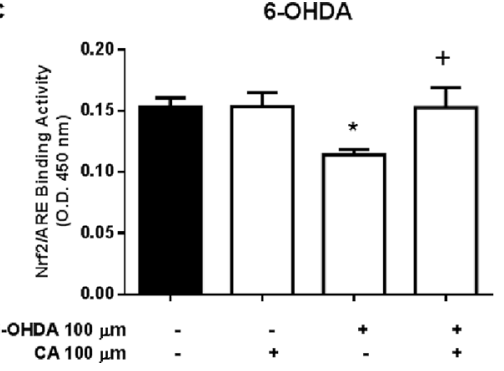

Figure 5.

Effects of caffeic acid (CA) on quinolinic acid (QUIN; a)-, ferrous sulfate $\left(\mathrm{FeSO}_{4}\right.$; $\left.\mathbf{b}\right)$ - or 6hydroxydopamine (6-OHDA; c)-induced changes in Nrf2/ARE binding activity in rat cortical slices. Values represent means \pm S.E.M. ( $\mathrm{n}=6$ experiments per group). ${ }^{*} P \unlhd \mathbf{0} .05$, different from the control; ${ }^{+} P \unlhd 0.05$, different from the toxin tested; two-way analysis of variance followed by post hoc Tukey's test. 

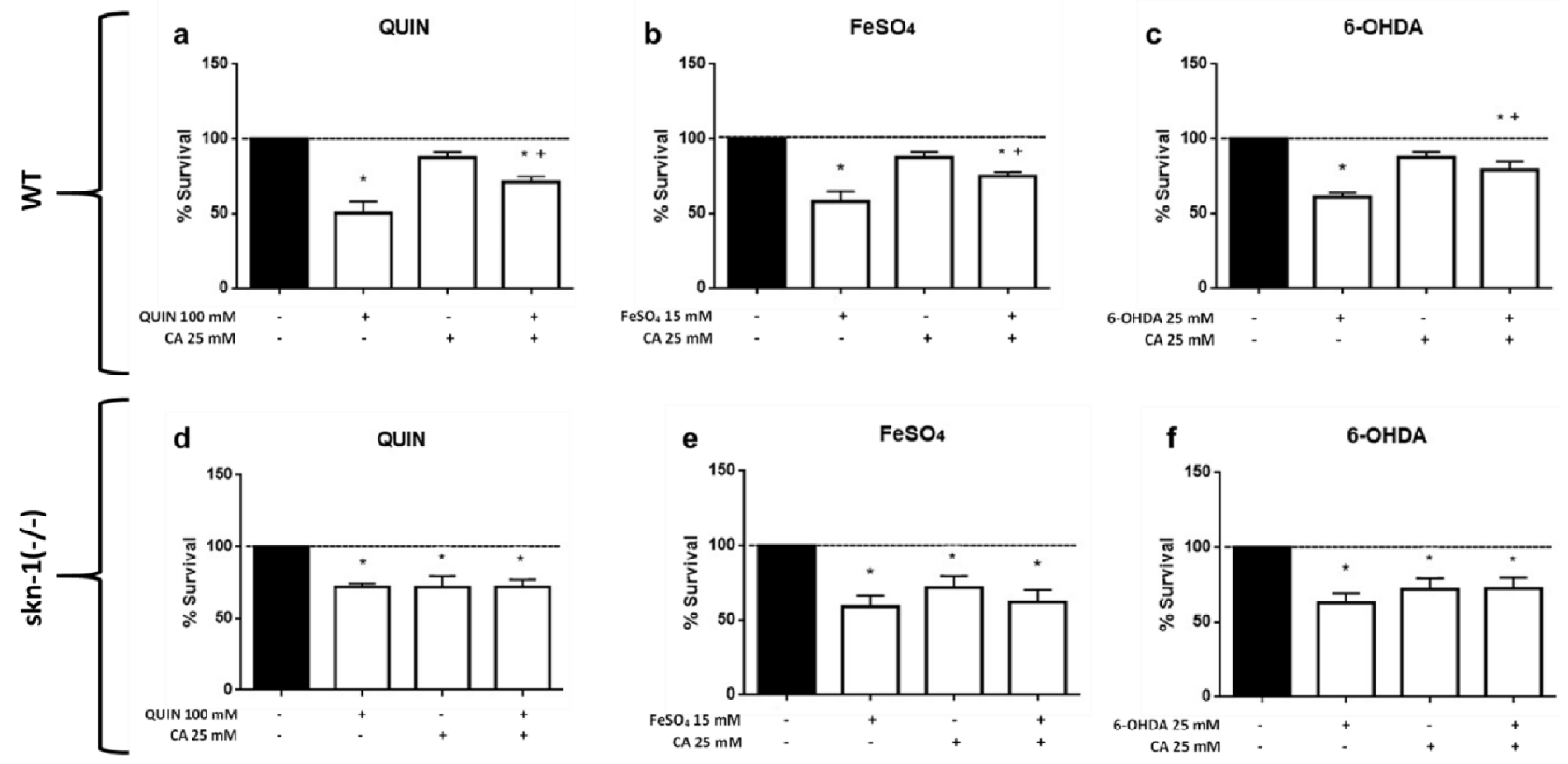

Figure 6.

Effects of caffeic acid (CA) on quinolinic acid (QUIN; a and $\mathbf{d})$, ferrous sulfate $\left(\mathrm{FeSO}_{4} ; \mathbf{b}\right.$ and e) or 6-Hydroxydopamine (6-OHDA; $\mathbf{c}$ and f)-induced changes in survival of $C$. elegans N2 [WT] and $s k n-1$ (ok2315) [skn-1-l-] strains. Values represent means \pm S.E.M. $(\mathrm{n}=5-7$ experiments per group). $* P \unlhd 0.05$, different from the control; ${ }^{+} P \unlhd 0.05$; different from the toxin tested; two-way analysis of variance followed by post hoc Tukey's test. 

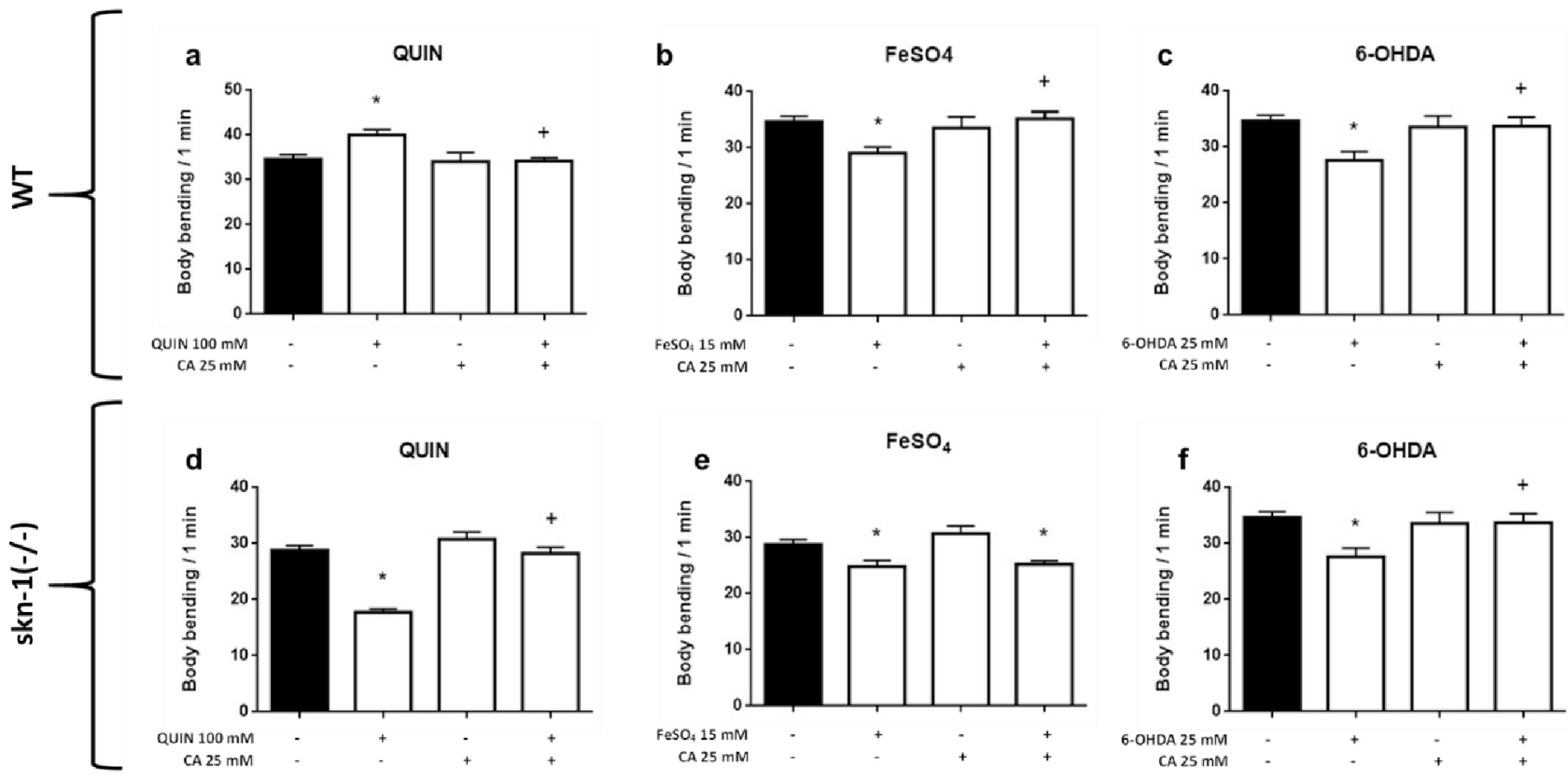

Figure 7.

Effects of caffeic acid (CA) on quinolinic acid (QUIN; $\mathbf{a}$ and $\mathbf{d})$, ferrous sulfate $\left(\mathrm{FeSO}_{4} ; \mathbf{b}\right.$ and $\mathbf{e}$ ) or 6-Hydroxydopamine (6-OHDA; $\mathbf{c}$ and $\mathbf{f}$ )-induced behavioural changes in $C$. elegans $\mathrm{N} 2$ [WT] and skn-1 (ok2315) [skn-1-/-] strains. Values represent means \pm S.E.M. $\left(\mathrm{n}=5-7\right.$ experiments per group). $\left.{ }^{*} P \unlhd\right) .05$, different from the control; $\left.{ }^{+} P \unlhd\right) .05$; different from the toxin tested; two-way analysis of variance followed by post hoc Tukey's test. 